\title{
Liquid Chromatography Particle Beam-Mass Spectrometry with Massive Cluster Impact*
}

\author{
Tao-Chin Lin Wang, Louis J. Cornio, and S. P. Markey \\ Section of Analytical Biochemistry, National Institute of Mental Health, National Institutes of Health, \\ Bethesda, Maryland, USA
}

\begin{abstract}
A new liquid chromatography-mass spectrometry technique is described that utilizes a particle beam interface to transport and deposit desolvated analyte molecules onto a target surface that is bombarded by a primary beam of massive multiply charged glycerol cluster ions to generate secondary ions for mass analysis. The massive cluster ion beam is generated by electrohydrodynamic emission from a solution of $1.5-\mathrm{M}$ ammonium acetate in glycerol. In the present instrumental configuration the massive cluster ion gun is placed above the target probe and the particle beam interface is connected through a side port of the mass spectrometer. The massive cluster ion beam and particle beam are intercepted by a target surface substituted for a conventional ion volume. The target surface is positioned such that it is $\sim 45^{\circ}$ to the primary cluster ion beam, the particle beam, and the mass analyzer axis. This geometric orientation represents a compromise among the performances of these three elements.

The feasibility of this liquid chromatography-particle beam on-line with massive cluster impact is demonstrated by flow injections of acetylcholine chloride and gramicidin S. Spectra generated from this preliminary study indicate promise for routine liquid chromatography-mass spectrometry of polar compounds by using a robust inlet and an effective generation of secondary ions without an added matrix. (f Am Soc Mass Spectrom 1996, 7, 293-297)
\end{abstract}

$\mathrm{T}$ There have been multiple instrumentation designs directed toward the development of routine liquid chromatography-mass spectrometry (LCMS) of polar organic compounds. To accomplish this goal, research has combined ionization techniques for nonvolatile samples with liquid chromatography (LC) interfaces compatible with the flow rate and solvent system of high-performance liquid chromatography applications. In recent years, electrospray ionization has extended LC-MS applicability to include large and polar biomolecules. However, the success of the electrospray process is largely dependent upon the composition of the sample solution and its flow rate. In this study, a new LC-MS technique that employs massive cluster impact (MCI) ionization [1] in combination with particle beam liquid chromatography is reported.

One feature that distinguishes massive cluster impact from conventional particle-induced desorption and ionization techniques, such as plasma desorption, fast-atom bombardment (FAB), or liquid secondary ionization mass spectrometry (LSIMS), is the identity

Address reprint request to: Dr. Sanford P. Markey, NIH/NIMH, Bldg. 10, Rm. 3D40, 10 Center Drive, MSC 1262, Bethesda, Maryland 20892-1262

* Part of this work was presented at the 43rd ASMS Conference on Mass Spectrometry and Allied Topics; Atlanta, Georgia, May 21-26, 1995. of the primary species. Massive cluster impact uses very large highly charged clusters of glycerol with an average of $10^{6}$ glycerol molecules and several hundred charges as the primary desorption beam. The primary beam is generated by electrohydrodynamic emission of electrolyte-glycerol solution in a vacuum $[1,2]$.

MCI mass spectrometric data indicate that heavy cluster ion beam bombardment is a promising soft ionization technique because it produces less fragmentation on the generated secondary ions than does a xenon primary beam [2]. A significant advantage of the $\mathrm{MCI}$ technique compared to FAB or LSIMS is the formation of singly and multiply charged molecular ions similar to electrospray ionization [3]. This greatly extends the applicable mass range from $\sim 3000 \mathrm{u}$ for static secondary ionization mass spectrometry to as high as $66 \mathrm{ku}$. Biomolecules that include phospholipids, carbohydrates, peptides, proteins, and oligonucleotides have been analyzed by this technique [4-6]. Second, Cornett et al. [7] recently reported that liquid matrices are not required for the successful detection of biomolecules by $\mathrm{MCI}$ ionization. $\mathrm{MCI}$ produces spectra from dissolved samples in commonly used FAB or LSIMS liquid matrices, for example, glycerol, thioglycerol, 3-nitrobenzyl alcohol, and dithiothreitoldithioerythritol, and from dry solid samples [2-7]. Finally, MCI has the ability to desorb the sample and 
generate ions without imposition of excessive contamination or radiation damage to the sample or matrix material. This advantage has been attributed to the high beam kinetic energy (on the order of $1 \mathrm{MeV}$ with $20-\mathrm{kV}$ acceleration voltage), but very low (less than $1 \mathrm{eV} /$ nucleon) specific energy $[2,3]$. Therefore, $\mathrm{MCI}$ appears to produce invariant mass spectra until the sample has been completely consumed [8].

The particle beam interface is an aerodynamically based sample-transporting device. An aerosol is generated from the liquid chromatograph (LC) effluent by He nebulization. Solvent evaporates rapidly in the desolvation chamber and is removed together with the dispersing gas in a momentum separator $[9,10]$. Essentially solvent-free analyte molecule particles are transported to the ion source and, in conventional electron ionization (EI) or chemical ionization (CI), flash vaporized in the ion source and ionized. Particle beam liquid chromatography-mass spectrometry has been shown to produce reference quality EI and CI mass spectra. However, its application range has been limited to analytes that show significant vapor pressure in these ion sources. Efforts to extend the applicable range to include compounds with low volatility under typical ion source conditions have included coupling particle beam transport with desorption ionization techniques such as FAB or LSIMS [11-15]. Conventional FAB and LSIMS matrices have been introduced into the system in several ways: application on a removable probe target, postcolumn or flow injection addition to the LC effluent, or introduction through a continuous flow $\mathrm{FAB}$ probe. The relative complexity of these techniques compared to electrospray-atmospheric pressure ionization inlets have not attracted interest in further development of particle beam-FAB or-LSIMS combinations.

Interfacing the LC particle beam with MCI combines the advantages of the particle beam technique as a routine LC-MS interface and MCI to extend the applicable mass range to include large nonvolatile thermally labile molecules. In this work, the feasibility of LC particle beam with MCI mass spectrometry was demonstrated by repetitive flow injections of acetylcholine chloride and gramicidin $S$ followed by an evaluation of this prototype system for future improvements.

\section{Experimental}

All the experiments were performed on a TSQ 70 (Finnigan-MAT, San Jose, CA) mass spectrometer equipped with a $\mathrm{MCI}$ ion gun (Phrasor Scientific, Inc., Duarte, CA). The original TSQ EI-CI ion source was used, but was inverted from its normal orientation to take advantage of a larger entrance aperture for the cluster ion beam. The filament block was removed from the TSQ source assembly. The MCI gun was positioned on a polymethyl methacrylate ( 0.5 in. thick) window in line with the EI electron entrance hole in the ion source. The solution used for cluster ion generation was $1.5-\mathrm{M}$ ammonium acetate in glycerol contained in a reservoir at atmospheric pressure. The glycerol solution was delivered through a stainless steel capillary emitter (2.8 in. long, 100- $\mu \mathrm{m}$ i.d.) to which high voltage is applied. Typical MCI gun operating voltages were $17 \mathrm{kV}$ on the cmitter and $15 \mathrm{kV}$ on the focus element. For stable emission current, the extractor voltage was set between 2 and $5 \mathrm{kV}$. The optimal signal stability was obtained when the cluster current monitor indicated between 4 and $10 \mu \mathrm{A}$. The flight tube for the cluster ion beam was extended to its maximum, approximately $1.5 \mathrm{in}$. away from the probe tip (Figure 1).

A particle beam interface (Hewlett Packard, Palo Alto, CA) delivered desolvated analyte molecules through a side port directly into an EI-CI ion source at room temperature as shown in the block diagram of Figure 2. The transfer line for the particle beam was extended such that it is locked into a side hole on the ion source that was intended in the original design for the gas chromatograph inlet. $\Lambda$ Hewlett Packard-1050 LC was used for sample delivery. The flow rate was set at $0.4 \mathrm{~mL} / \mathrm{min}$ and the solvent was $1: 1$ water and methanol. Flow injections of $1-\mu \mathrm{L}$ sample solution were performed by a Hewlett-Packard autosampler. For the gramicidin S (Sigma Chemical Co., St. Louis, MO) experiments, 0,15 , or $50 \mathrm{ppm}$ of glycerol were added to the water.

A polished copper probe tip was positioned $\sim 45^{\circ}$ to the incidence of the cluster primary beam, the incoming particle beam, and the mass analyzer axis. The position and angle of the tip were then carefully adjusted for maximum sensitivity. The probe tip was

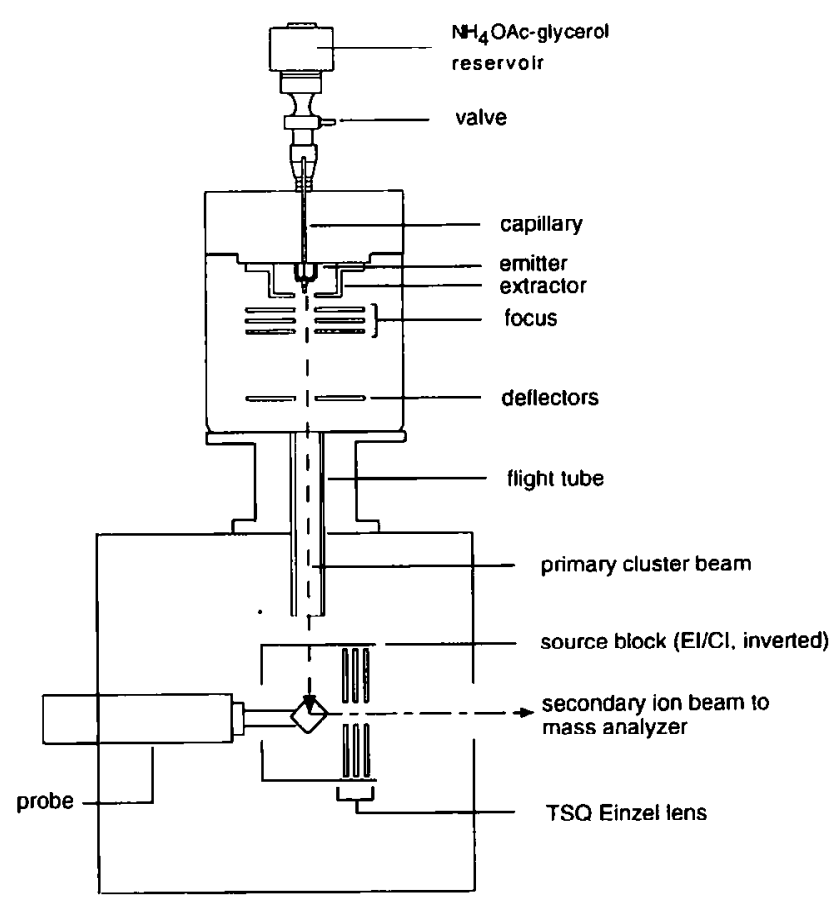

Figure 1. Schematic diagram of $\mathrm{MCI}$ ion source. 


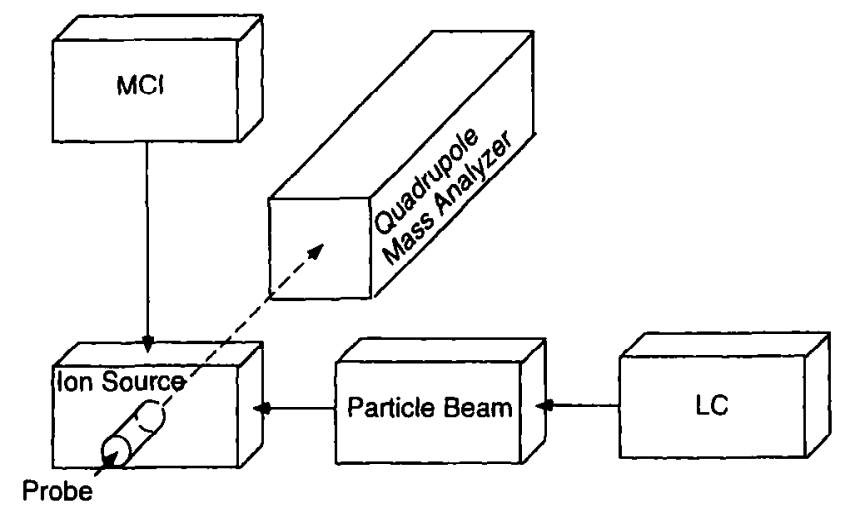

Figure 2. Block diagram of particle beam LC-MS with $\mathrm{MCI}$.

approximately four times larger in surface area than the Finnigan standard FAB or LSIMS copper tip.

\section{Results and Discussion}

The feasibility of liquid chromatography particle beam $\mathrm{MCI}$ was demonstrated by repetitive flow injections of $20,50,100$, and $200 \mathrm{ng}$ of acetylcholine chloride. Acetylcholine was chosen because of its low molecular weight and volatility, as well as its ionic character. A selected ion monitoring (SIM) experiment of acetylcholine $\left(\mathrm{M}^{+}\right)$at $m / z 146$ is shown in Figure 3. The ion chromatogram of several injections contains peaks with 30-s width and consistent height. This result indicates that desolvated acetylcholine molecules were transported by the particle beam to the target surface and desorbed from the surface by the massive cluster primary beam in a very short period of time. The response was linear $\left(r^{2}=0.996\right)$ in the $20-200 \mathrm{ng}$ per injection range as shown in the insert of Figure 3 . The lower peak intensity observed at $m / z 146$ for 20 -ng injections of acetylcholine chloride could be due to sample loss in the transport process. The background contains glycerol characteristic debris ions from $\mathrm{MCI}$

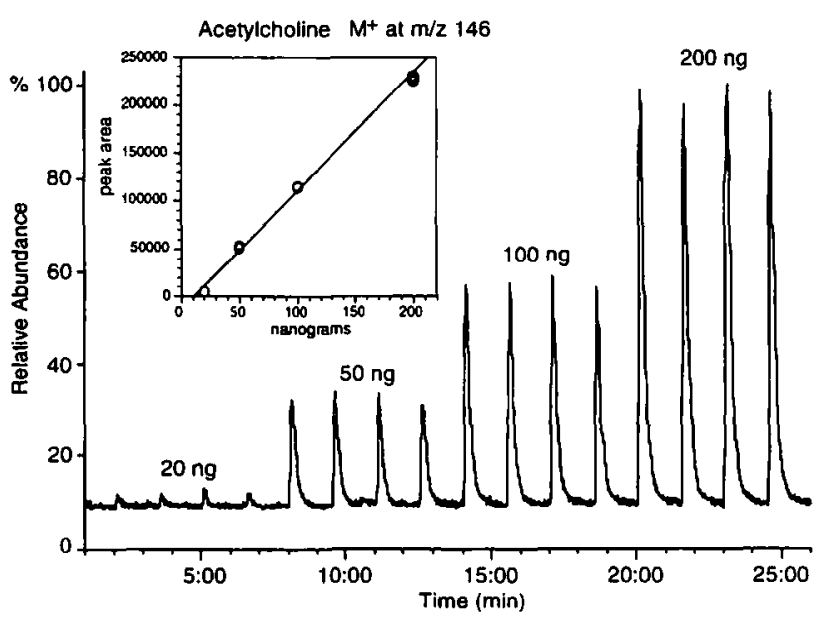

Figure 3. Flow injections of 20-, 50-, 100-, and 200-ng acetylcholine chloride. Selected ion monitoring of $m / z 146$. The insert shows a linear response curve in this range. (e.g., $m / z$ 93, 110, 185, 202, etc.) that remain constant as shown in Figures 4 and 5 . The corresponding spectra obtained from the sample signal and the background are shown in Figure 5. The molecular ion from acetylcholine $\mathrm{M}^{+}$at $m / z \quad 146$ and a fragment ion at $m / z 87\left[\mathrm{CH}_{2}-\mathrm{CH}_{2}-\mathrm{N}^{+}-\left(\mathrm{CH}_{3}\right)_{3}\right]$ along with choline $\left(\mathrm{M}^{+}\right.$ at $m / z$ 104) are observed.

Liquid chromatography particle beam-mass spectrometry with $\mathrm{MCI}$ was tested next with a larger molecule, gramicidin S. Gramicidin S is a cyclic decapeptide antibiotic with a molecular weight of 1141 . Figure 6 shows repetitive flow injections of $500 \mathrm{ng}$ of gramicidin $\mathrm{S}$ observed in the scan mode of acquisition and Figure 7 shows the full scan mass spectrum obtained from the signals in Figure 6. Clearly, molecules as large as gramicidin $\mathrm{S}$ are able to be transported by the particle beam, desorbed, and mass analyzed. It was found that mixing glycerol in the water portion of the mobile phase improved the stability and sensitivity of detection. In this experiment $7.5 \mathrm{ppm}$ of glycerol were added in the mobile phase. Figure 8 shows the sensitivity of the present instrumental configuration by repetitive injections of $50 \mathrm{ng}$ of gramicidin S. Sample detections were performed by SIM acquisition of the +2 charge state of the molecule (i.e., $[\mathrm{M}+2 \mathrm{H}]^{2+}$ at $\mathrm{m} / z$ 571). In this experiment $25 \mathrm{ppm}$ of glycerol were added to the mobile phase. The consistency in peak intensity and background demonstrates that stable signals are obtained.

These results permit several preliminary observations:

1. MCI spectra may be generated with a LC particle beam interface by providing a suitable collection surface in a region of ion extraction into a mass spectrometer.

(a) Acetylcholine m/2:146

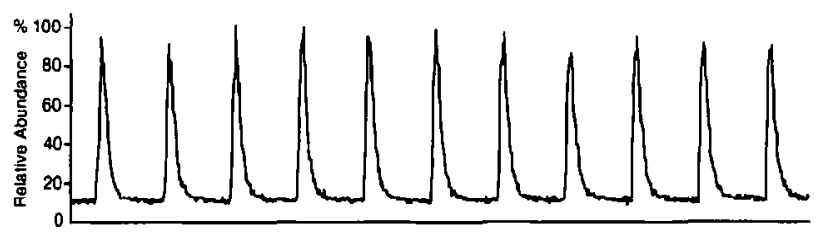

(b) $\mathrm{MCl}$ debris ion $\mathrm{mz}: 110$

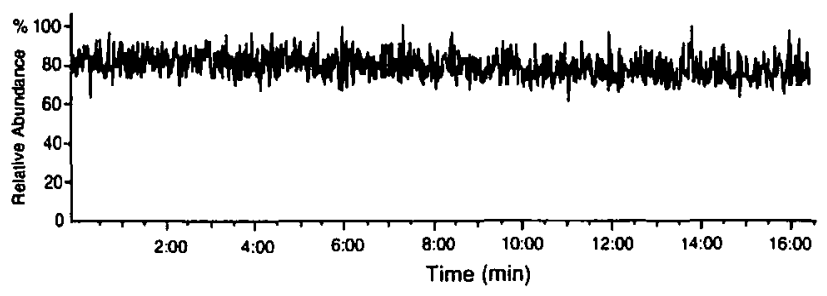

Figure 4. (a) Flow injections of $200 \mathrm{ng}$ of acetylcholine chloride. Data were acquired in the scan mode with mass-to-charge ratio range from 80 to 220 . (b) The background remains constant as shown by the characteristic debris ion $m / z 110$ chromatogram from MCI. 

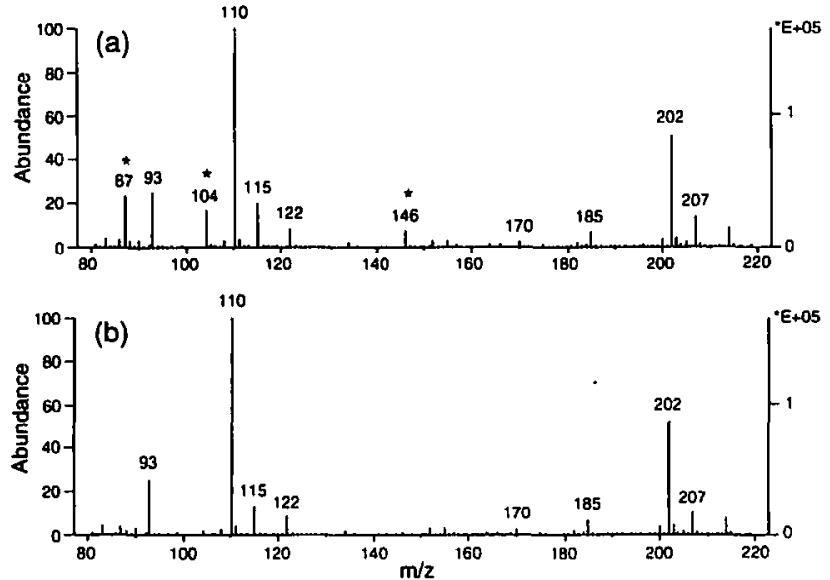

Figure 5. Spectra obtained from (a) acetylcholine and (b) background signals in Figure 4. $\mathrm{MCl}$ debris ions, for example, 93, 110,185 , and 202 remain constant, while the molecular ion from acetylcholine at $m / z 146$ and a fragment ion at $m / z \quad 87$ along with choline $(m / z 104)$ are observed.

2. LC solvent flow rates in the range typical for EI-CI particle beam-mass spectrometry are compatible with $\mathrm{MCI}$ spectral generation.

3. Normal static FAB or LSIMS matrices can be added into the LC effluent at very low concentrations to improve signal stability and sensitivity.

4. Because the solvent is removed very efficiently in the interface, the success of LC particle beam MCI spectrometry should be independent of solvent type (e.g., acetonitrile, hexanes, isopropanol, methanol, water, etc.).

5. LC particle beam with MCI has the potential of providing routine LC-MS detection of polar organic compounds and peptides.

We have observed that there are additional parameters that affect the optimal operation of MCI: these include the spray needle characteristics, flow rates of

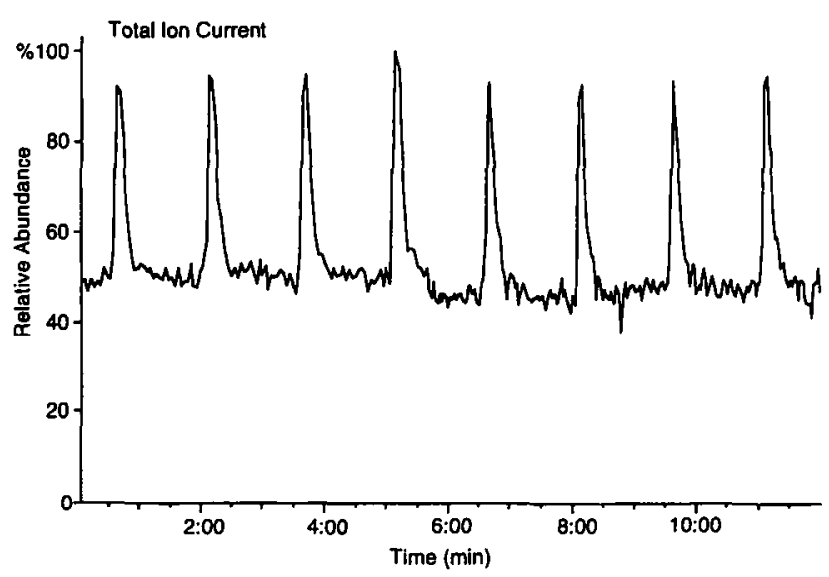

Figure 6. Flow injection of $500 \mathrm{ng}$ of gramicidin $\mathrm{S}$ with 7.5-ppm glycerol added to the moble phase. Data were acquired in the scan mode with mass-to-charge ratio range from 500 to 1200 and 3-s duration per scan.

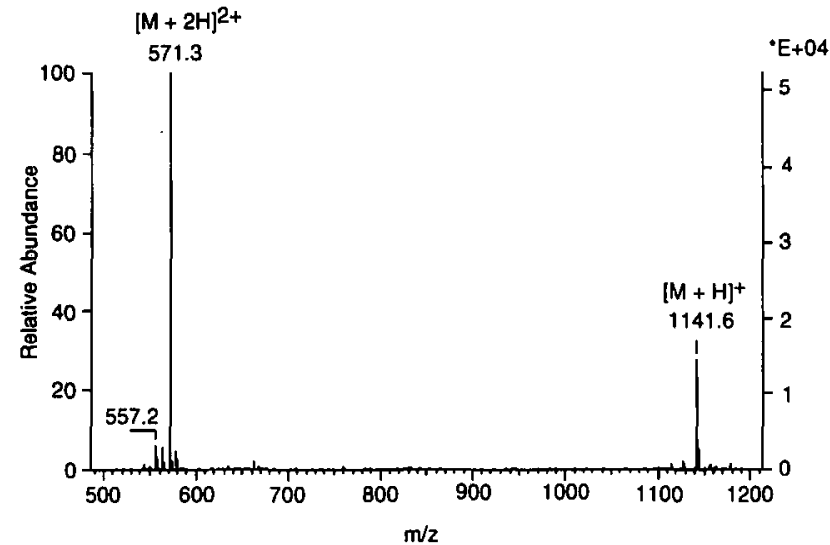

Figure 7. Gramicidin S full scan spectrum obtained from signals in Figure 6.

the glycerol solution, and potential difference between the source capillary emitter and the extractor. For example, sharpening the spray needle significantly improves the spray process and thus increases the stability of the primary cluster beam. Glycerol solution flow rates and the potential between the emitter and extractor control the size of the glycerol clusters generated and alter the cluster distribution, thus affecting the desorption and ionization efficiency of the analyte. The glycerol solution flow rate, in turn, is controlled by the viscosity of the solution and spray needle diameter and length. Glycerol solution viscosity can be controlled by the electrolyte concentration and temperature of the solution.

Experimental results have shown that the yield of the secondary ions is highly dependent upon the relative position and angle of the probe tip to the $\mathrm{MCI}$ primary beam, particle beam, and the mass analyzer. Ideally, we would like to have both the MCI cluster ion beam and the particle beam in line with the mass analyzer axis and aimed at a target surface area optimal for efficient secondary ion extraction. However, it is almost impossible to accomplish this ideal configuration in practice because the physical sizes of the

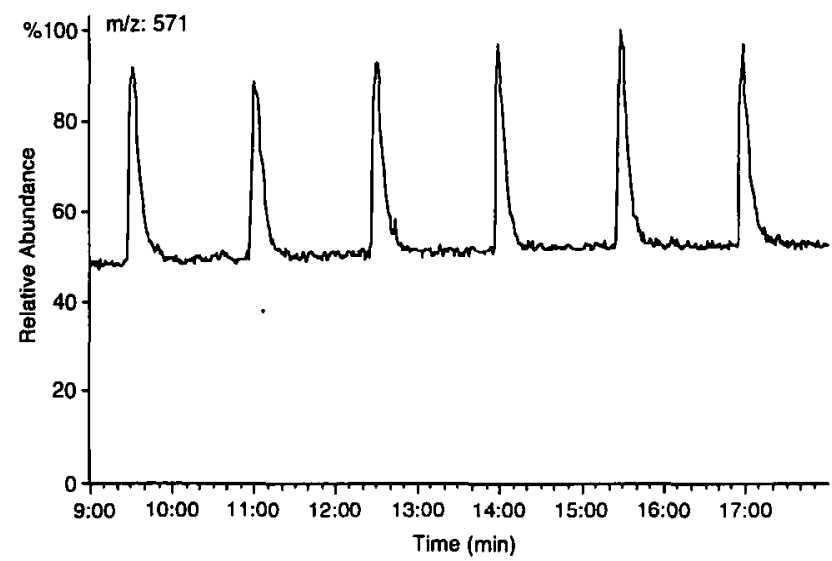

Figure 8. Flow injections of $\mathbf{5 0} \mathrm{ng}$ of gramicidin S. Data were acquired in the SIM mode of the +2 charge state of gramicidin S. 
components necessitate a compromise configuration. Future efforts will include optimization of the geometric positions of MCI gun, particle beam and target surface in order to achieve better sensitivity.

It is known that the addition of a carrier like ammonium acetate (either pre- or postcolumn) enhances the transport of analytes through the particle beam [16]. We observe an improvement in signal detection stability and sensitivity after the addition of low concentrations of glycerol in the mobile phase. It is not clear whether this improvement is due to a carrier effect during sample transport process, a matrix effect in the desorption and ionization process, or a combination of both effects.

\section{Conclusion}

It has already been indicated in previous reports [2-4, 7-8] that MCI has advantages as a softer ionization method compared to FAB or LSIMS in producing singly and multiply charged molecular ions with less fragmentation and surface damage. As a particle-induced desorption and ionization technique, its success depends largely upon the quality, that is, size, stability, energy, and distribution of the primary cluster ion beam, but is independent of the means employed to transport samples to the target surface. From our preliminary results, coupling the liquid chromatography particle beam-mass spectrometry with MCI shows promise as a robust technique for mass analysis of polar organic molecules. With further research dedicated to the improvement of its detection limit and signal stability, we anticipate this combination will become a routine and rugged LC-MS device.

\section{Acknowledgments}

The authors wish to thank Peter Todd of Oak Ridge National Laboratory, John Mahoney of Phrasor Scientific, and Carl ljames of National Institutes of Mental Health for many helpful discussions and suggestions on the optimal operation of the gun. The authors also wish to thank Phrasor Scientific for extended loan of the $\mathrm{MCl}$ equipment.

\section{References}

1. Mahoney, J. F.; Perel, J.; Ruatta, S. A.; Martino, P. A.; Husain, S.; Lee, T. D. Rapid Commun. Mass Spectrom. 1991, 5, 441.

2. Mahoney, J. F.; Perel, J.; Lee, T. D.; Legesse, K. Int. J. Mass Spectrom. Ion Processes 1987, 79, 249.

3. Mahoney, J. F.; Comett, D. S.; Lee, T. D. Rapid Commun. Mass Spectrom. 1994, 8, 403.

4. Fabris, D.; Wu, Z.; Fenselau, C. C. J. Mass Spectrom. 1995, 30, 140.

5. Dookeran, N. N.; Todd, P. Proceedings of the 36th ASMS Conference on Mass Spectrometry and Allied Topics; Atlanta, Georgia, May 1995; $\mathrm{p} 1154$.

6. Dookeran, N. N.; Todd, P. Proceedings of the 36th ASMS Conference on Mass Spectrometry and Allied Topics; Atlanta, Georgia, May 1995; p 1191.

7. Cornett, D. S.; Lee, T. D.; Mahoney, J. F. Rapid Commun. Mass Spectrom. 1994, 8, 996.

8. McMahon, J. M.; Dookeran, N. N.; Todd P. J. Am. Soc. Mass Spectrom. 1995, 6, 1047.

9. Willoughby, R. C.; Browner, R. F. Anal. Chem. 1984, 56, 2626.

10. Winkler, P. C.; Perkins, D. D.; Williams, W. K.; Browner, R. F. Anal. Chem. 1988, 60, 489.

11. Kirk, J. D.; Browner, R. F. Proceedings of the 36th ASMS Conference on Mass Spectrometry and Allied Topics; San Francisco, California, June 1988; p 1233.

12. Willoughby, R. C.; Buchner, J. D.; Sheehan, E. W. Proceedings of the 36th ASMS Conference on Mass Spectrometry and Allied Topics; San Francisco, California, June 1988; p 1297.

13. Kirk, J. D.; Browner, R. F. Biomed. Environ. Mass Spectrom. 1989, 18, 355.

14. Little, J. L.; Cook, L. A.; Perry, R. L.; Tindall, G. W.; Vestal, M. Proceedings of the 38th ASMS Conference on Mass Spectrometry and Allied Topics; Tucson, Arizona, June 1990; p 1019.

15. Sanders, P. E. Rapid Commun. Mass Spectrom. 1990, 4, 123.

16. Bellar, T. A.; Behymer, T. D.; Budde, W. L. J. Am. Soc. Mass Spectrom. 1990, 1, 92. 